\title{
Lidil
}

Revue de linguistique et de didactique des langues

\section{Un exemple de prédicats complexes opérant sur des prédicats simples}

Les conjonctions de subordination du français, de l'espagnol et de l'italien

Mireille Piot

\section{CpenEdition}

\section{Journals}

Édition électronique

URL : http://journals.openedition.org/lidil/2694

DOI : 10.4000/lidil.2694

ISSN : 1960-6052

Éditeur

UGA Éditions/Université Grenoble Alpes

\section{Édition imprimée}

Date de publication : 1 juin 2008

Pagination : 115-132

ISBN : 978-2-84310-124-3

ISSN : 1146-6480

Référence électronique

Mireille Piot, « Un exemple de prédicats complexes opérant sur des prédicats simples », Lidil [En ligne], 37 | 2008, mis en ligne le 01 septembre 2009, consulté le 19 avril 2019. URL : http://

journals.openedition.org/lidil/2694; DOI : 10.4000/lidil.2694

Ce document a été généré automatiquement le 19 avril 2019

(C) Lidil 


\section{Un exemple de prédicats complexes opérant sur des prédicats simples}

Les conjonctions de subordination du français, de l'espagnol et de l'italien

\section{Mireille Piot}

1 Dans l'optique transformationnelle harrissienne du 'lexique-grammaire' correspondent à la fonction d'élément prédicatif tous les éléments lexicaux qui sélectionnent les arguments d'une phrase simple et peuvent être considérés comme la tête de cette phrase. Dans cette catégorie, on retrouve donc non seulement de manière classique les classes de verbes (non auxiliaires), mais aussi les classes d'adjectifs caractérisés par la propriété No est Adj et de manière moins attendue les classes de substantifs associés à une classe d'auxiliaires verbaux particuliers, les verbes supports : tous ces éléments jouant le même rôle de noyau de la phrase simple.

2 On peut considérer également dans le cadre harrissien étendu à la grammaire des opérateurs que les conjonctions de subordination (désormais Conjs) constituent une classe particulière de super-prédicats, les opérateurs sur des opérateurs (0oo), qui opèrent sur les prédicats des phrases simples qu'ils conjoignent. Nous nous attacherons ici à décrire un certain nombre de phénomènes qui illustrent cette analyse en prenant des exemples dans le domaine des conjonctions de subordination du français (et/ou de l'espagnol et/ou de l'italien) qui montrent en particulier que les propriétés des phrases conjointes et notamment de la seconde (introduite par la conjonction), dans une moindre mesure pour la première (ou principale), sont des propriétés transmises par l'item conjonctif à ces phrases.

3 Nous envisagerons ainsi d'abord, dans ces trois langues, le cas des conjonctions « finales" ou de «but» qui présentent la particularité d'imposer des contraintes à la fois sur la nature du sujet de la principale, ainsi que sur le mode puis sur le temps de la subordonnée; ces contraintes sont à mettre au compte des constructions de base des substantifs sur lesquels sont formées ces conjonctions.

4 Les conjonctions "hypothético-conditionnelles", ensuite, imposent des contraintes en temps-mode à la subordonnée qu'elles introduisent (ou aspectuelles à son équivalent 
nominal), ce qui est particulièrement visible en français si l'on considère celles qui sont formées à partir d'un substantif comme cas/coup/fois par rapport aux conjonctions «temporelles» formées des mêmes substantifs avec pour immédiate différence formelle la nature de la détermination accompagnant ces substantifs dans l'une ou l'autre de ces classes.

Notre troisième exemple concerne les conjonctions appartenant à la classe des " additives-exceptives", composée donc de ces deux sous-classes, qui sont formées sur des formes prépositionnelles, adverbiales ou participiales. L'appartenance à l'une ou l'autre sous-classe de cette classe de conjonctions déclenche un comportement particulier vis-à-vis de la présence de la négation dans les phrases qu'elles conjoignent, comportement qui apparait étroitement lié au sémantisme de ces items mais aussi à la nature syntaxique de l'opération en cause. Ces phénomènes sont également observables dans les trois langues.

6 De fait, ces super-prédicats au sein d'une phrase complexe se comportent de manière analogue aux prédicats fonctionnant en phrase simple: ils sélectionnent la nature sémantique et les propriétés de construction de leurs arguments, en l'occurrence ici représentés par les phrases sous la conjonction.

\section{La classe des Conjs «finales » ou de « but »}

Cette classe a pour caractéristiques principales :

8 1) une contrainte sur la nature du sujet de la principale : obligatoirement «humain » comme le montrent les exemples :

(1) a. (Pedro/*el viento) sopló fuertement, (con el objeto/para) que sea avivado el fuego

b. (Pierre/*le vent) a soufflé violemment, (dans le but/pour) que le feu soit attisé

c. (Pietro/*il vento) soffio con forza, (affinché/con lo scopo che) sia attizzato il fuoco.

\section{Remarque :}

Cette contrainte Nhum obligatoire est respectée, bien sûr, non seulement dans des phrases telles que :

Les tapis sont imprégnés d'une solution (afin de/pour) les détacher

Las alfombras están impregnados de solución (con el objeto de/para) quitarles las manchas

I tappeti sono impregnati di solvente (con lo scopo di/per) smacchiarli

11 ou encore :

L'eau monte dans le tube pour équilibrer le mercure

El agua está subiendo por el tubo (con el objeto de/para) equilibrar el mercurio

L'acqua sta saliendo nel tubo (con lo scopo di/per) equilibrare il mercurio

où sont respectivement à l'œuvre les transformations [passif] ou [-moyen]; mais aussi dans l'exemple :

Une voiture attend les voyageurs pour les conduire au lac

Un coche está esperando a los viajeros para conducirles al lago

Una macchina sta aspettando $i$ viaggiatori per portarli ai laghi

qui n'est interprétable que comme :

On attend les voyageurs (avec/dans) une voiture pour les conduire au lac

(Se/Alguien) está esperando a los viajeros con un coche para conducirles al lago

(Si/Cualcuno) sta aspettando i viaggiatori con una macchina per portarli ai laghi

14 ce que prouve a contrario l'inacceptabilité, en revanche, de : 
*Mon vélo m'attend en bas pour me conduire au lac

* Mi bici me está esperando abajo para conducirme al lago

* La mia bicicleta mi sta aspettando giù per portarmi ai laghi Psubj) comme le montrent :

(2) a. Pedro sopló fuertemente, (con el objeto/para) que (sea/*es) avivado el fuego

b. Pierre a soufflé violemment, (dans le but/pour) que le feu (soit/*est) attisé

c. Pietro soffiò con forza, (affinché/con lo scopo che) (sia/*è) attizzato il fuoco.

la complétive est susceptible d'une réduction automatique à l'infinitif ( $V$-inf) obligatoirement sous la condition d'identité des sujets de la subordonnée et de la principale :

(3) a. (A fin/En la intención) de quedarse (sola/*solo), Maria prefiere alejar a Pedro

b. (Afin/Dans le dessein) de rester (seule/*seul), Marie préfere éloigner Pierre

c. (Affine/Nello scopo) di rimanere (sola/*solo), Maria preferisce allontanare Pietro.

4) Enfin, l'insertion des adverbes dans la subordonnée (complétive ou infinitive) montre que le temps de celle-ci est obligatoirement le futur (le présent et le passé étant exclus) même dans le cas où la forme du verbe correspond à une forme du passé :

(4) a. (Con el objeto de/para) que sea avivado el fuego (mañana/*ayer), Pedro sopló

fuertemente

b. (Pour/Dans le but) que le feu soit attisé (demain/*hier), Pierre a soufflé violemment

c. (Affinché / con lo scopo che) il fuoco sia attizzato, (domani/*ieri), Pietro soffiò con forza

(5) a. (Para / Con el propósito de) haberlo acabado (mañana/*ayer), Maria trabaja mucho

b. (Pour / Dans le dessein d') avoir fini cela (*hier/?*en ce moment/demain), Marie travaille

beaucoup

c. (Per/Nell'intenzione di) averlo finito (domani/*ieri), Maria lavora molto

En effet, le temps relève toujours obligatoirement du « futur » malgré la forme 'passé' du verbe.

Or, toutes ces propriétés sont celles des constructions définitionnelles des $N$ « appropriés » formants des Conjs appartenant à cette classe qui sont :

(Pierre/*le vent) a (pour/le) (but/intention/dessein/...) d'attiser le feu, en soufflant 
(Pietro/*Il vento) ha (l'intenzione/la finalità/...) di attizzare il fuoco, soffiando.

Vs

(7) a ?*Hay un (objeto/propósito/...) para avivar el fuego.

$b$ ?*Il y a un (but/dessein/intention) pour attiser le feu

$c ?^{*} C$ 'è (un'intenzione/una finalità) per attizzare il fuoco ;

2) la contrainte de mode est également observée, avec le subjonctif obligatoire pour la subordonnée :

(8)a. (Pedro/*El viento) tiene el (objeto/propósito/...) que el fuego sea avivado, al soplar

b. (Pierre/*le vent) a (pour/le) (but/intention/dessein/...) que le feu soit attisé, en soufflant

c. (Pietro/*Il vento) ha (l'intenzione/la finalità/...) che il fuoco sia attizzato, soffiando

3) la réduction à l'infinitive correspondante a lieu uniquement sous condition d'identité des sujets de la subordonnée et de la principale :

(9) a. Al apartar a Pedro, Maria tiene (el fin/la intención) de quedarse (sola/*solo)

b. Marie a (le/pour) (but/dessein) de rester (seule/*seul), en éloignant Pierre

c. Maria ha (l'intenzione/lo scopo) di rimanere (sola/*solo), allontanando Pietro

30 4) enfin est également observée la contrainte de temps obligatoirement futur pour la subordonnée attachée à ces $\mathrm{N}$ « appropriés » comme le montre l'insertion des adverbes de temps :

(10) a. (Pedro/*El viento) tiene el (objeto/propósito/...) de avivar el fuego (mañana/*ayer), al soplar

b. (Pierre/*le vent) a (pour/le) (but/intention/dessein/...) d'attiser le feu (demain/*hier), en soufflant

c. (Pietro/*Il vento) ha (l'intenzione/la finalità/...) di attizzare il fuoco (domani/*ieri), soffiando...

Le comportement en tous points analogues des phrases conjointes par ces Conjs, de même classe syntactico-sémantique, comme des $\mathrm{N}$ 'appropriés' sur lesquelles elles sont construites dans les trois langues, montre à l'évidence le rôle crucial, pour la syntaxe comme pour la sémantique de ces phrases complexes, de ces joncteurs en fonction de super-prédicats.

Il s'agit donc ici du premier exemple que nous donnons en faveur de l'analyse de ces Conjs qu'a proposée Z.S. Harris $(1976,1977)$, pour l'anglais notamment.

\section{La classe des conjonctions « hypothético- conditionnelles »}

Cette autre classeest caractérisée par l'existence de contraintes de type aspectuel qu'elles imposent à leur subordonnée : qu'il s'agisse de la sélection des temps-modes du verbe, ou bien de la nature de la détermination du $N$ subordonné. Ces contraintes sont transmises par les substantifs constituants de ces Conjs particulières, notamment: hypothèse, condition, réserve, etc.Nous rendons compte de ces contraintes définitionnelles pour toute cette classe, avant de nous pencher sur ce qui différencie celles formées sur les substantifs cas et coup de leurs homonymes appartenant à la classe des « temporelles » qui n'imposent pas le même type de contraintes sur leur subordonnée. 


\section{Les contraintes aspectuelles définitionnelles de la classe des " hypothético-conditionnelles »}

\section{Les contraintes de temps-mode sur le verbe de la subordonnée}

Lorsque la subordonnée phrastique est introduite par une Conjs qui permet une alternance où/que $P$ pour introduire la subordonnée, les temps possibles sont futur, conditionnel ou subjonctif :

(11) a. En la hipótesis de que (acabes/acabaría/acabará), te (doy/daría/daré) un premio

b. Dans l'hypothèse (où tu (finiras/finirais)/que tu finisses), je te récompense(rais(s))

c. Nell'ipotesi (in cui/che) (finisca/finiresti/finirai), ti. premierò...

Avec des Conjs où seul est possible l'introducteur que, les temps sont généralement futur et subjonctif, plus rarement conditionnel :

(12) a. Te (doy/daría/daré) un premio, a condición de que (acabes/acabaría/acabará) ese trabajo esta noche

b. Je te récompense(rais(s)), à (la) condition que tu (finisses/finiras) ce travail ce soir

c. Ti premierò, a condizione che (finisca/finiresti/finirai) questo lavoro stasera.

\section{Les contraintes sur la détermination du $\mathrm{N}$ subordonné}

Ces Conjs, comme en général toutes celles construites sur des substantifs ou des prépositions ${ }^{1}$, permettent une complémentation nominale parallèle à la subordonnée phrastique décrite immédiatement ci-dessus. Cette complémentation nominale ne connait généralement aucune restriction quant à la nature possible de sa détermination. Tel est le cas avec les Conjs appartenant à la classe des « causales » (par exemple du fait de/ por el hecho de/dal fatto), des «temporelles" (par exemple au moment de/en el momento de/ nel momento), des « finales » (par exemple dans le but de/en el objeto de/con lo scopo). On a, en effet, en parallèle à :

(13) a. Por el hecho de que (él/el tren) ha llegado tarde, Pedro esperaba en la estación

b. Du fait qu'(il/le train) est arrivé tardivement, Pierre attendait dans la gare

c. Dal fatto che (lui/il treno) non è arrivato in tempo, Pietro aspettava nella stazione

(14) a. En el momento en que trasladaron a Maria, Pedro estaba en Paris

b. Au moment où Marie était évacuée, Pierre était à Paris

c. Nel momento in cui Maria stava spostata, Pietro stava a Parigi

(15) a. En el objeto de que llegue pronto, Pedro llamó a Pablo

b. Dans le but qu'il arrive rapidement, Pierre a appelé Paul

c. Con lo scopo che arrivi pronto, Pietro ha chiamato Paolo

les possibilités de paraphrases nominales (respectivement (16) à (18)) sans restriction sur leur détermination :

(16) a. Por el hecho de su llegada tarde, Pedro esperaba en la estación

b. Du fait de ((son/une) arrivée/l'arrivée du train) tardive, Pierre était à la gare

c. Dal fatto del arrivo (del treno) non in tempo, Pietro aspettava nella stazione

(17) a. En el momento de (l/su) traslado, Pedro estaba en Paris

b. Au moment de (sa/une/l') évacuation, Pierre était à Paris

c. Nel momento del (suo) trasferimento, Pietro stava a Parigi

(18) a. En el objeto de (una/su) llegada rápida, Pedro llamo a Pablo

b. Dans le but de (son/une/l') arrivée prochaine, Pierre a préparé un état des lieux

c. Con lo scopo de (uno/el suo) arrivo veloce, Pietro ha chiamato Paolo.

En revanche, les conjonctions appartenant à cette classe d'«hypothéticoconditionnelles", transmettent à la complémentation nominale une contrainte 
aspectuelle sur sa détermination (parallèle à celle de temps-mode sur le verbe de la subordonnée phrastique, cf. (19) à (21)). On a ainsi en parallèle à :

(19) a. A condición de que me ayudes, te daré un premio

b. À condition que tu m'aides, je te récompenserai

c. A condizione che mi aiuti, ti premierò

(20) a. En la hipótesis de que venga con rapidez, podremos salir esta noche

b. Dans l'hypothèse qu'il vienne rapidement, on pourra sortir ce soir

c. Nell'ipotesi che venga prontissimo, potremmo uscire stasera

(21) a. En el caso de que fluctúe el franco, se preverá medidas

b. Dans le cas que le franc fluctue, on prévoira des mesures

c. Nel caso che oscilli il franco, si prevera misure

39 les paraphrases nominales respectives :

(22) a. A condición de una ayuda tuya, te daré un premio

b. À condition d'une aide de ta part, je te récompenserai

A condición ne de un tuo aiuto, ti premierò

(23) a. En la hipótesis de una llegada suya rápida, podremos salir esta noche

b. Dans l'hypothèse d'une venue rapide de sa part, on pourra sortir ce soir

c. Nell'ipotesi d'una sua venuta veloce, potremmo uscire stasera

(24) a En el caso de una fluctuación del franco, se preverá algunas medidas

$b$ Dans le cas d'une fluctuation du franc, on prévoira quelques mesures

c Nel caso di una oscillazione del franco, si preverá alcune misure,

mais non les paraphrases qui seraient celles normalement attendues et qui sont observées ci-dessus avec les autres classes de Conjs :

(25) a. *A condición de tu ayuda, te daré un premio

b. *A condition de ton aide, je te récompenserai

c. ${ }^{*} A$ condizione del tuo aiuto, ti premierò

(26) a. En la hipótesis de su llegada rápida, podremos salir esta noche

b. *Dans l'hypothèse de sa venue rapide, on pourra sortir ce soir

c. ${ }^{*}$ Nell'ipotesi della sua venuta veloce, potremmo uscire stasera

(27) a. *En el caso de la fluctuación del franco, se preverá algunas medidas

b. *Dans le cas de la fluctuation du franc, on prévoira quelques mesures

c. *Nel caso de la oscillazione del franco..., si prevera alcune misure.

41 C'est-à-dire que les déterminants de nature définie (Ddéf ou Poss) sont exclus en présence des Conjs de cette classe, et qu'il existe donc une contrainte impérative que nous avons signalée en ce cas comme Dét = un oblig. Or, l'on sait depuis les travaux de Guillaume notamment (1919) que l'expression de l'indéfinitude correspond à celle d'une modalité aspectuelle.

\section{Le cas des conjonctions « hypothético-conditionnelles » vs « temporelles » formées sur les mêmes substantifs cas, coup, fois en français.}

Nous devons distinguer ici deux situations :

Celle d'une part des Conjs «hypothético-conditionnelles » les plus figées formées sur fois et coup : (des/quelque) fois, un coup dont l'indice de figement nous est fourni par le choix de l'introducteur que pour la subordonnée ; en effet, la variante où n'est pas permise, d'où les contrastes :

(28) a. (Des/Quelque) fois qu'il pleuvrait, je prends mon parapluie

b. *(Des/Quelque) fois où il pleuvrait, je prends mon parapluie 
(29) a. Un coup qu'il viendrait, on ira à la pêche

b. *Un coup où il viendrait, on ira à la pêche.

En regard, les Conjs «temporelles » formées sur les mêmes substantifs (avec possibilité d'autres déterminants pour fois) autorisent naturellement cette alternance :

(30) a. (A) (chaque/toutes les) fois (que/où) Pierre vient, il pleut

b. Un(e) (coup/fois) (que/où) Pierre était là, on a beaucoup ri.

Et la situation du substantif cas qui autorise l'alternance où/que $e^{2} P$ aussi bien pour les «temporelles »:

(A/dans) (chaque/tous les) cas (où/que) Pierre vient, il pleut

que pour les « hypothético-conditionnelles»:

(Dans/pour) le cas (que/où) il pleuvrait, prends donc ton parapluie.

Ici, la différenciation manifeste entre les deux classes correspond respectivement à l'absence vs la présence de contraintes en temps-mode sur la subordonnée, même si s'y adjoignent parfois des différenciations dans la détermination (par exemple quantifieur universel vs Dét défini) de ces substantifs ou au niveau des formes de l'introducteur de la subordonnée.

\section{Les conjonctions « additives-exceptives » et la négation}

Les deux sous-classes composant cette classe particulière de Conjs présentent une différence d'ordre syntactico-sémantique dans leur comportement vis-à-vis de la négation.

En effet, avec les 'exceptives' l'une seulement des deux phrases conjointes (la principale ou la subordonnée) doit obligatoirement comporter une 'négation' (ou un élément négatif), mais non les deux, comme le montrent les paires de contrastes suivants :

(33) a. Pedro hará todo, salvo (que no hará) la limpieza

Pierre fera tout, sauf (qu'il ne fera pas) le menaje

Pietro fara tutto, salvo (che non fara) la pulizia

b. *Pedro hará todo, salvo (que hará) la limpieza

*Pierre fera tout, sauf (qu'il fera) le ménage

*Pietro fara tutto, salvo (che fara) la pulizia

(34) a. Pedro no hará nada, salvo (que hará) la limpieza

Pierre ne fera rien, sauf (qu'il fera) le ménag Pietronon fara niente, salvo (che non fara) la

pulizia

b. *Pedro no hará nada, salvo (que no hará) la limpieza

* Pierre ne fera rien, sauf (qu'il ne fera pas) le ménage

*Pietro non fara niente, salvo (che non fara) lapulizia

En revanche, la sous-classe des «additives» exclut la présence de Nég dans l'une seulement des phrases conjointes, et ne permet la conjonction de ces phrases que si toutes deux sont à la fois : soit assertives, soit négatives, ce que montrent par exemple les paires de contrastes suivants :

(35) a. Además (que habla) de su trabajo, Pedro habla de su vida Outre (qu'il parle) de son travail, Paul parle de la vie

Oltre (che parla) del suo lavoro, Paolo parla della sua vita

b. *Además (que no habla) de su trabajo, Pedro habla de su vida

* Outre (qu'il ne parle pas) de son travail, Paul parle de la vie

* Oltre (che non parla) del suo lavoro, Paolo parla della sua vita

(36) a. Además (que no habla) de su trabajo, Pedro no habla de su vida

Outre (qu'il ne parle pas) de son travail, Paul ne parle pas de la vie 
Oltre (che non parla) del suo lavoro, Paolo non parla della sua vita

b. * Además (que no habla) de su trabajo, Pedro habla de su vida

* Outre (qu'il parle) de son travail, Paul ne parle pas de la vie

* Oltre (che non parla) del suo lavoro, Paolo non parla della sua vita ${ }^{3}$.

Dans l'un comme dans l'autre cas, ces contraintes d'ordre syntactico-sémantique sont imposées par chacune de ces sous-classes de Conjs aux phrases conjointes. Évidemment, le sémantisme de ces items est à chaque fois en cause mais pas seulement, comme le montre un phénomène intéressant que nous avons découvert.

51 En effet, un certain nombre d'items appartenant à la sous-classe des "exceptives " peuvent passer dans la sous-classe des «additives », moyennant la perte des contraintes sur la présence obligatoire de la négation dans l'une des deux phrases conjointes. Toutes les « exceptives » ne le peuvent pas. Par exemple, les plus 'récentes' ( $\mathrm{xvI}^{\mathrm{e}}$ siècle) comme excepté et sauf ne peuvent autoriser la perte de cette contrainte et le passage dans l'autre sous-classe. Alors que le peuvent les plus anciennes (IX $\mathrm{x}^{\mathrm{e}}$ siècle) : hormis, à part, hors, etc. qui sont 'spatiales'd'origine (comme l'« additive » outre). On a ainsi les contrastes :

(37) a. Aparte de mi maleta, tomaré mi ordenador

(A part/Hormis) ma valise, je prendrai mon ordinateur

= (A part/Hormis) [le fait que je prendrai] ma valise, je prendrai mon ordinateur

A parte la mia valigia, pigliaro il mio computer

b. *(Excepto/salvo) mi maleta, tomaré mi ordenador

*(Excepté/sauf) ma valise, je prendrai mon ordinateur

*(Eccetto/salvo) la mia valigia, pigliaro il mio computer.

Notre analyse de ce dernier phénomène nous pousse à considérer que les plus anciennes de ces Conjs 'exceptives' montrent dans cet emploi un processus de grammaticalisation plus avancé que les dernières dont le contenu lexical univoque et donc plus explicite est sans doute par ailleurs également en cause dans le contraste observé.

Les quelques exemples que nous apportons ici à l'appui de notre thèse pourraient être multipliés : ils ne sont pas isolés, ni ne constituent des exceptions. Nous n'avons choisi que quelques-uns parmi les phénomènes, de nature assez diverse comme nos exemples le démontrent, qui sont susceptibles de montrer le rôle de ces connecteurs sur les phrases qu'ils conjoignent dans trois des principales langues romanes.

À travers ces quelques exemples empruntés (parmi beaucoup d'autres) à nos travaux de longue date sur les conjonctions de subordination du français et un peu plus récemment sur l'espagnol et l'italien, nous espérons avoir ici illustré et conforté l'analyse avancée par Z.S. Harris (depuis 1976, 77 et jusqu'à sa mort) de ces connecteurs comme de superprédicats transmettant leurs propriétés et leurs possibilités de construction aux phrases sur lesquelles ils opèrent. Ces propriétés s'avèrent dans chaque cas mettre en relation la syntaxe et la sémantique à travers les choix lexicaux opérés. 


\section{BIBLIOGRAPHIE}

GUILLAUME, G. (1919) : Le problème de l'article et sa solution dans la langue française, Paris, Hachette ;

rééd. 1975, Paris, Nizet.

HARRIS, Z.S. (1976) : Notes du cours de syntaxe, Paris, Le Seuil.

HARRIS, Z.S. (1978) : Operator Grammar of English. In Linguisticae Investigationes, T.2, vol. 1, 55-92.

HARRIS, Z.S. (1982) : A Grammar of English on Mathematical Principles, New York, JohnWiley and Sons.

HARRIS, Z.S. (1988) : Language and Information, New York, Columbia University Press.

HARRIS, Z.S. (1991) : A Theory of Language and Information : A Mathematical Approach, Oxford

University Press, USA.

РІОТ, M. (1978) :Études transformationnelles de quelques classes de conjonctions de subordination du français. Thèse de $3^{\mathrm{e}}$ cycle, Université Paris 7 et LADL ; $455 \mathrm{p}$.

PIOT, (1979) : Les conjonctions « finales » du français, Lingua e Stile, I,Bologna, 39-60.

PIOT, (1995) : Composition transformationnelle de phrases par subordination et par coordination.

Thèse d'état ès Lettres et Sciences humaines, Université Paris 7 et LADL, 426 p. ; 1998 : Le Septentrion, collection 'Thèse à la carte'.

PIOT, (2003) : L'expression dela 'cause', de la 'finalité' et de la conséquence : la conjonction pour et ses équivalents en français, espagnol et italien, synchronie-diachronie, in P. Blumenthal et J.-E. Tyvaert (éds), La cognition dans le temps, 121-134, collection “Linguistische Arbeiten",Tübingen, Niemeyer.

PIOT, (2005) : Sur les fausses prépositions : 'sauf' et 'excepté', Journal of French Language Studies, 15.3, 297-314.

\section{Corpus en ligne}

Frantext (Cnrs, Atilf), Abu (Cnam)

CORDE

Corpus diacrónico del español, Madrid, Real academia española [2005-2006-2007] : Banco de datos CREA

Corpus de referencia del español actual, Madrid, Real academia española[2005-2006-2007]

Ovitalnet

Vocabolario del Italiano e del Italiano Antico (gattoweb) [2005-2006].

\section{NOTES}

1. Que nous analysons comme obtenues par effacement de l'un des substantifs "appropriés » à telle ou telle classe. 
2. La forme que dans les exemples où est permise l'alternance avec où a une allure archaïque ou régionale.

3. Un certain nombre de ces exemples indiqués comme impossibles peuvent être autorisés, mais alors sans les réductions d'éléments que nous y avons indiquées comme facultatives. L'interprétation est alors que e.g. parler de son travail et ne pas parler de sa vie (ou inversement) sont posés par le locuteur comme positivement équivalents dans son esprit.

\section{RÉSUMÉS}

Dans cet article, nous illustrons à partir de phénomènes empruntés à l'espagnol, le français et l'italien la thèse de Z.S. Harris analysant les conjonctions de subordination comme une classe de super-prédicats (la classe des 000 ) opérant sur les prédicats simples, noyau des phrases simples. Nous abordons différents types de phénomènes qui permettent de mettre en évidence cette fonction particulière des items conjonctifs.

The aim of this paper is to analyse phenomena drawn from complex sentences in French, Italian and Spanish, in order to demonstrate Z.S. Harris's thesis concerning the classification of subordinating conjunctions as a class of super-predicates (the Ooo class) operating on simple predicates, the core of simple sentences. Different examples will be drawn on in order to highlight this specific function of conjuncitve items. 